\title{
Amphibienreste aus dem Oberpliozän von Kaltensundheim (Rhön, Thüringen)
}

\author{
Gottfried Böhme ${ }^{1}$ \\ Mit 13 Abbildungen

\section{Zusammenfassung}

Aus den oberpliozänen Füllsedimenten eines fossilen Erdfalles bei Kaltensundheim in der Rhön (Thüringen) werden Skelettreste von anuren Amphibien beschrieben. Es liegen Reste von Bufo bufo (Linnaeus, 1758), Bufo sp., Rana temporaria Linnaeus, 1758 sowie vermutliche Parietale nicht bestimmbarer Salientia vor. Die bisher mehrfach genannte Latonia lässt sich nicht nachweisen. Sie muss aus der Faunenliste von Kaltensundheim gestrichen werden.

Schlüsselwörter: Amphibia, Salientia, Bufo, Rana, Mitteleuropa, Pliozän.

\begin{abstract}
Skeletal remains of anuran amphibians are described from sediment filled earthfall depression of upper Pliocene age near Kaltensundheim/Rhön (Thuringia). Bufo bufo (Linnaeus, 1758), Bufo sp., Rana temporaria Linnaeus, 1758, as well as a probable parietal of an indeterminate salientian could be distinguished. There is no evidence of the often mentioned Latonia. This genus should be eliminated from the faunal list of Kaltensundheim.
\end{abstract}

Key words: Amphibia, Salientia, Bufo, Rana, Central Europe, Pliocene.

\section{Einleitung}

Aus den Füllsedimenten eines fossilen Erdfalls aus dem Oberpliozän wurden durch systematische Grabungen zahlreiche Skelettreste von Wirbeltieren geborgen, darunter auch Reste von Amphibien. Diese wurden bereits mehrfach erwähnt (Böhme 1968, 1992, 2000, Sanchiz 1998), jedoch bisher noch nicht beschrieben und mit Abbildungen vorgestellt. Das Fundgut ist Eigentum des Naturhistorischen Museums Schleusingen (NHMS), Thüringen, als Nachfolgeeinrichtung der Naturwissenschaftlichen Abteilung der Meininger Museen.

\section{Die Fundstelle}

Der fossile Erdfall liegt NW des Dorfes Kaltensundheim in der Rhön (Kreis Schmalkalden/Meiningen, Thüringen) am rechten Hang des LotteBaches. Lage und Gestalt des Erdfalles wurden durch Peilstangenuntersuchungen, Schürfe und
Geländebeobachtungen erkundet (Schaarschmidt 1958; Böhme 1968, 1992). Sie ergaben für ihn einen nahezu kreisförmigen Umriss mit ca. 100 m Durchmesser. Nach den ersten Zufallsfunden fossiler Wirbeltierreste als Folge von Schachtarbeiten für eine Wasserleitung wurden in den Jahren 1958 (F. Schaarschmidt), 1962-66 (G. Böhme) und 1976-78 (H.-D. Kahlke) systematische Probennahmen und Grabungen in den Füllsedimenten durchgeführt.

Die Füllsedimente des Erdfalles bestehen aus grauschwarzen, gebänderten mergeligen Tonen und Schluffen, die in limnischem Milieu mit fluviatilen Einflüssen sedimentiert wurden. Die ungestörte Böschungsschichtung fällt in den durch die Grabungen erschlossenen Bereichen mit ca. $35^{\circ}$ zum Zentrum des Erdfalles ein. Durch subaquatische Rutschungen kam es z. T. zu erheblichen Stauchungen und Faltungen des Sediments und der darin eingebetteten Wirbeltierfossilien.

Eine besondere Bedeutung hat die Fundstelle durch die Überlieferung vollständiger, noch teilweise artikulierter Skelette von Wirbeltieren

\footnotetext{
1 Museum für Naturkunde, Institut für Paläontologie, Invalidenstraße 43, D-10115 Berlin, Germany. Erhalten April, angenommen Juni 2002
} 
(z.B. Mastodonten und Hirsche). Es handelt sich einerseits um Elemente einer Waldfauna (Säugetiere), für die der frühere Erdfallsee zur tödlichen Falle wurde, andererseits um Elemente einer Fauna, für die der See Lebensraum oder Teil desselben war (Fische und Amphibien). Bisher wurde nur ein Teil der überlieferten Fauna deskriptiv publiziert (z.B. Böhme 1963). Die Skelettreste von Amphibien sollen im Folgenden vorgestellt werden.

\section{Zur Altersstellung}

Eine Alterseinstufung der Füllsedimente in das „Oberpliozän“ ermöglichen unter den bisher geborgenen Faunenresten lediglich die Skelettfunde des Mastodonten Mammut (Zygolophodon) borsoni (Hays 1834) und des Palaeolaginen $\mathrm{Hy}$ polagus sp. Eine detailliertere stratigraphische Einstufung versuchten Krutzsch (1988) anhand der Mikroflora sowie Mai \& Walther (1988) auf der Grundlage von karpologischen Resten aus den Füllsedimenten. Nach der Auffassung von Krutzsch handelt es sich um eine „kühl gemäBigte Minimum-Flora von DBF-Charakter" (deciduous broadleaved forest) mit vielen sogenannten quartären Kräuterelementen und repräsentiert die Basis des Villafranchiums (Minimum zwischen Reuver s. str. und Berga, ca. 2,6-2,7 my). Die von Mai \& Walther untersuchte Makroflora soll dagegen die „erste echte intraoberpliozäne Verarmungs- und Abkühlungsphase an der Basis des Oberpliozäns" belegen. Sie wird von diesen Autoren mit dem Florenkomplex von Ceyssac im Französischen Zentralmassiv mit einem Alter von ca. 3,4-3,8 my gleichgesetzt.

\section{Skelettreste von Amphibien}

Reste von Amphibien wurden erstmals bei der Wiederaufnahme systematischer Grabungen an der Fundstelle im November 1962 geborgen. Es handelte sich zunächst um eine polyspezifische Knochenkonzentration innerhalb des Sedimentkörpers ohne erkennbare Skelettverbände. Diese erbrachte jedoch den reichsten Fundkomplex an Kleinwirbeltierresten aus dieser Fundstelle (Fundkomplex Kals. 858 mit Skelettresten von mindestens 3-4 Individuen; NHMS-MTe 976). Später wurden so reiche Funde nicht mehr geborgen. In den Folgejahren wurden aber weitere Amphibienreste gefunden, darunter artiku- lierte Teilskelette (Fundplan bei Böhme 1968: Anl. 13).

\section{Taxonomischer Teil}

Amphibia Linnaeus, 1758

Salientia Laurenti, 1768

Bufonidae Gray, 1825

Bufo Laurenti, 1768

Es liegen fragmentarische Skelettelemente mehrerer Individuen vor (NHMS-MTe 976-978). Nur wenige kompakte Elemente sind vollständig überliefert (Prämaxillare, Scapula, Wirbel, Ischium). Die unterschiedlichen Dimensionen einzelner Elemente, insbesondere des Extremitätenskeletts, belegen die Existenz verschiedener Alterstufen. Spezifische Merkmale sind bei der Gattung Bufo vor allem an Frontoparietale, Humerus, Sacrum und Ilium ausgebildet (Böhme 1977). Die Gestaltung der Humeri zeigt, dass sowohl Reste von $\hat{\delta} \hat{\sigma}$ als auch von sind. Für die Artbestimmung ist die Morphologie der Frontoparietale von entscheidender Bedeutung. Die vorliegenden Frontoparietale unterscheiden sich in der Ausbildung der dorsalen Fläche grundlegend. Daraus ergibt sich die Existenz von mindestens zwei Bufo-Arten im Fundgut von Kaltensundheim. Die Frontoparietalia können jedoch nicht eindeutig den anderen Skelettelementen zugeordnet werden.

\section{Bufo bufo (Linnaeus, 1758)}

Abb. 1, 5, 9, 11, 12

Zahlreiche Skelettelemente aus mehreren Fundkomplexen (NHMS-MTe 976-978).

Frontoparietale: 1 sin. (Abb. 1), 1 dex. Frontoparietale und Prooticooccipitale nicht verbunden, dorsale Fläche ohne Ornamentierung, glatt, incrassatio frontoparietalis zugespitzt eiförmig, zugespitzte Seite laterat gerichtet.

Praemaxillare: wie bei der rezenten Art.

Sacrum: das fragmentarische Sacrum zeigt die für die rezente Art typischen Merkmale.

Ilium (Abb. 11, 12): 2 sin., 3 dex. Die Dimensionen der Ilia belegen mittelgroße Individuen. Sie liegen jedoch z.B. im oberen Bereich der metrisch untersuchten umfangreichen jungquartären Bufo bufo-Population von Pisede (Böhme 1982).

Scapula (Abb. 5): 3 sin., 1 dex., wie bei der rezenten Art. 
$1 \mathrm{~A}$

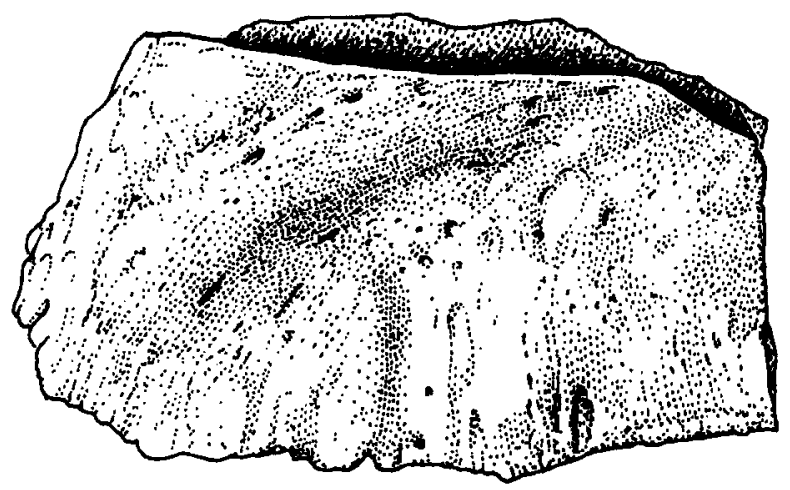

1B

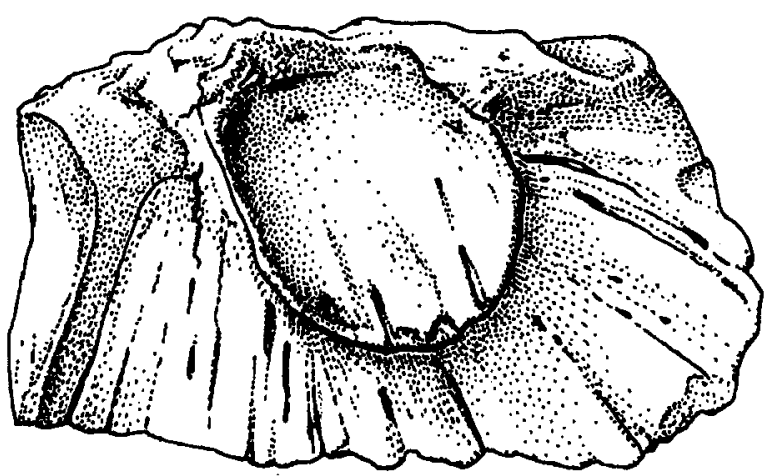

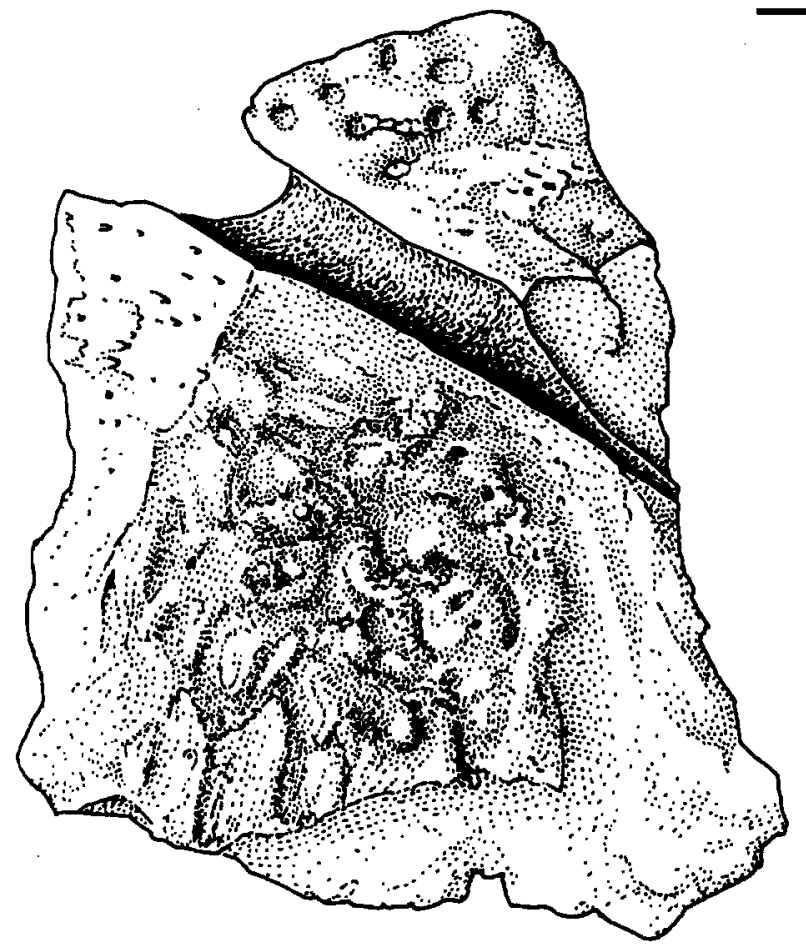

2A

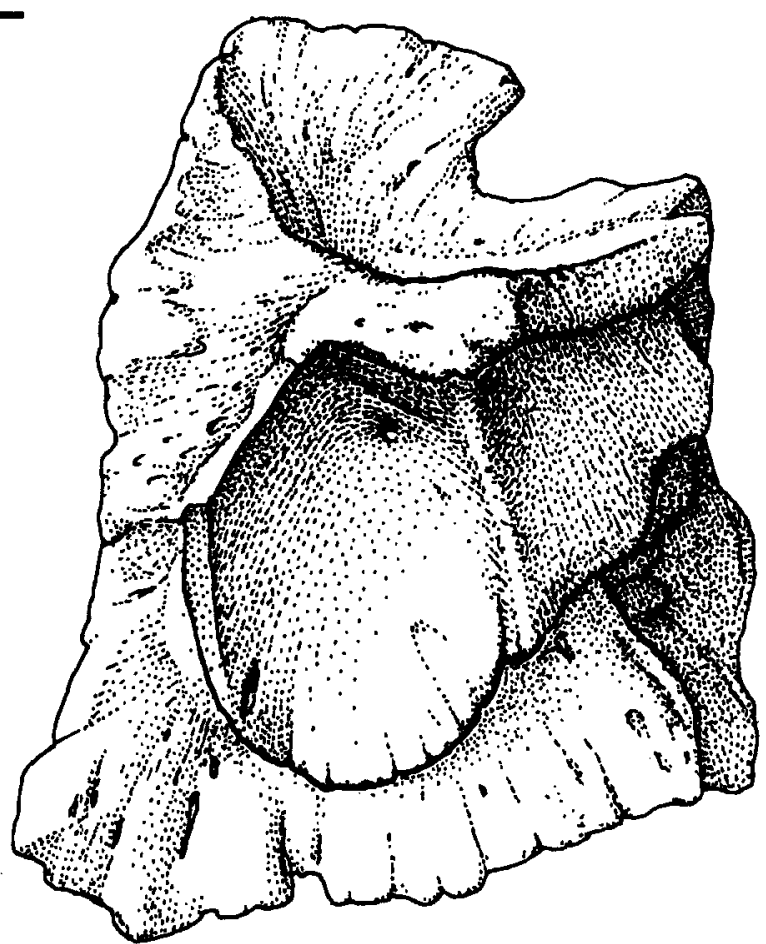

2B

Abb.1-2. 1. Bufo bufo, Frontoparietale sin. (NHMS-MTe 976/6); 2. Bufo sp., Frontoparietale dex. (NHMS-MTe 976/2); A, von dorsal; B, von ventral. Für diese und alle folgenden Abbildungen Maßstabbalken $=1 \mathrm{~mm}$.

Figs 1-2. 1. Bufo bufo, left frontoparietal (NHMS-MTe 976/6); 2. Bufo sp., right frontoparietal (NHMS-MTe 976/2); A, dorsal view; B, ventral view. In this and all following figures scale bar $=1 \mathrm{~mm}$.

Humerus (Abb. 9): wie bei der rezenten Art.

Neben Extremitätenresten mittelgroßer Individuen, die etwa der Durchschnittsgröße mitteleuropäischer Exemplare entsprechen, liegen aus dem Fundkomplex Kals. 858 auch sehr robuste Skelettelemente zweier Individuen vor (Prämaxillare, unbezahnte Maxillare, Wirbel, Scapula, of

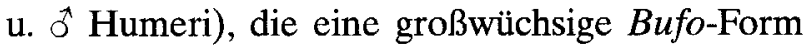
belegen (KR-Länge $>10 \mathrm{~cm}$ ). $\mathrm{Ob}$ es sich dabei um eine weitere Bufo-Art oder nur um ein besonders großes Individuum von Bufo bufo bzw. eine großwüchsige Supspezies handelt, kann nicht entschieden werden. Die Merkmale der großen Skelettelemente stimmen weitgehend mit denen von Bufo bufo überein. Bei der rezenten Art Bufo bufo sind solche großwüchsigen Formen mit besonderen äußeren Merkmalen und mediterraner Verbreitung als Subspezies B. bufo spinosus (Daudin, 1803) von der Nominatform abgegrenzt worden.

Humerus (Abb. 7 O, Abb. 8 o): Es liegen die Fragmente beider Humeri eines männlichen und eines weiblichen Individuums vor, die gleiche $\mathrm{Di}$ mensionen erreichen. Der epicondylus ulnaris erreicht bei $\delta \sigma^{\star}$ die Höhe der eminentia capitata; 

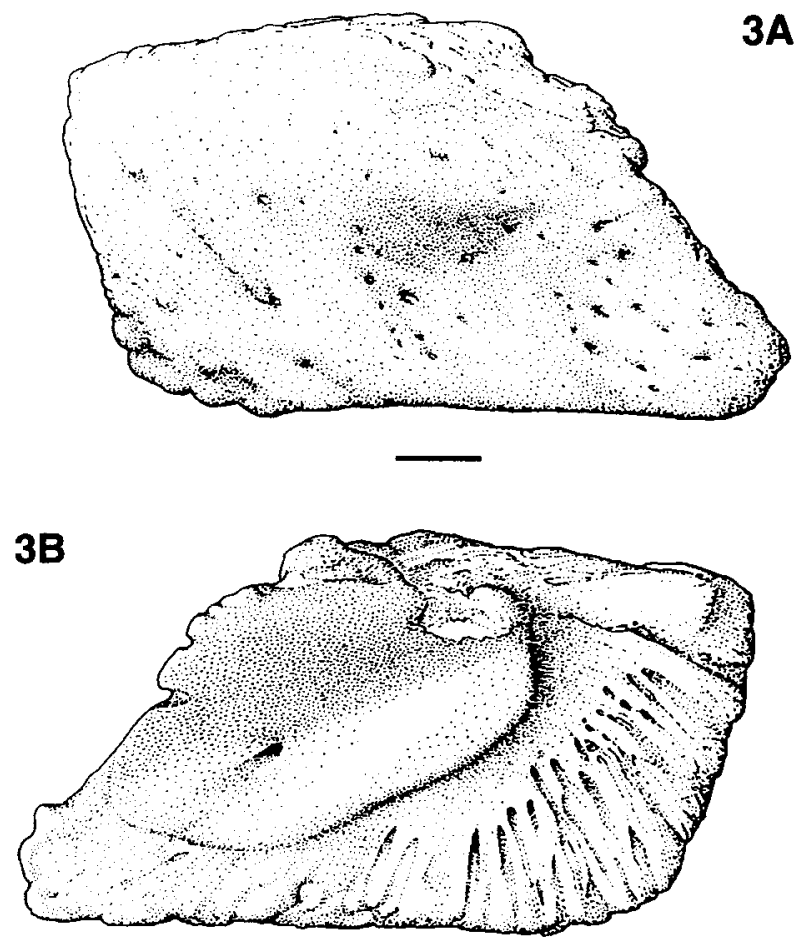

Abb. 3. Salientia indet., Parietale (NHMS-MTe 976/1); A. von dorsal; $\mathbf{B}$, von ventral.

Fig. 3. Salientia indet., parietal (NHMS-MTe 976/1); A, dorsal view; B, ventral view.

epicondylus radialis schwächer; crista medialis bis etwa zur halben Humerus-Länge kräftig entwickelt, crista lateralis erreicht nur etwa $2 / 3 \mathrm{der}$ Länge der crista medialis, crista ventralis nur im Ansatz erhalten.
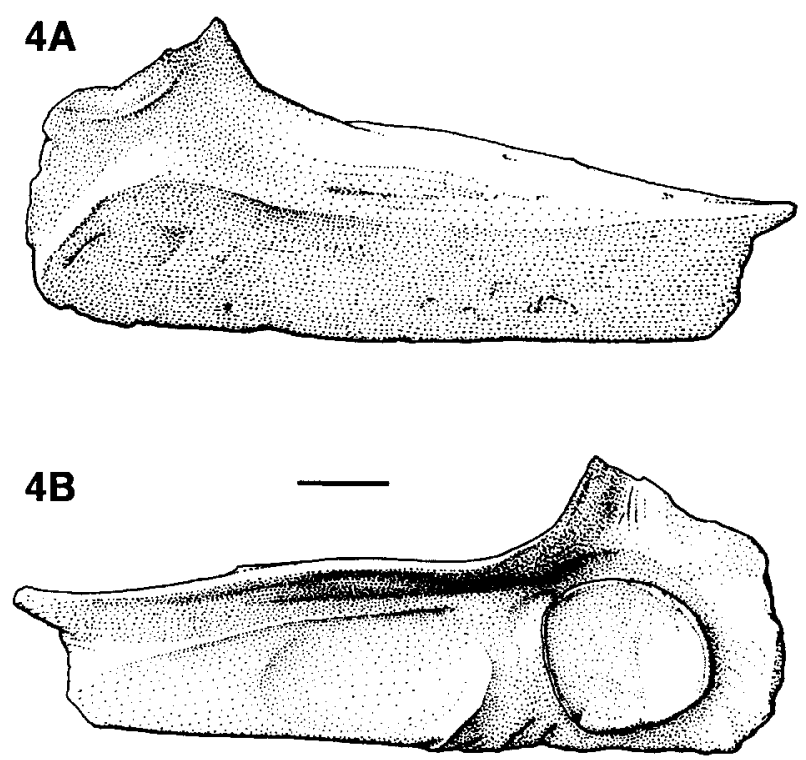

Abb. 4. Rana temporaria, Frontoparietale sin. (NHMS-MTe 978/1); $\mathbf{A}$, von dorsal; $\mathbf{B}$, von ventral.

Fig. 4. Rana temporaria, left frontoparietal (NHMS-MTe 978/1); A, dorsal view; B, ventral view.
Scapula (Abb. 6): Es liegen eine Scapula sin. und das Fragment einer Scapula dex. vor. Die crista anterior ist kräftig entwickelt, die margo anterior kräftig rugos und anterior gewölbt.

\section{Bufo sp. indet.}

Abb. 2

Frontoparietale + Prooticooccipitale (NHMS-MTe 976/2): Frontoparietale paarig, mit Prooticooccipitale verbunden, dorsale Oberfläche des Frontoparietale kräftig rugos ornamentiert, die incrassatio frontoparietalis leicht zugespitzt eiförmig, zugespitzte Seite laterat gerichtet. Der frontale Abschnitt ist nicht überliefert.

Möglicherweise gehört das ornamentierte Frontoparietale und Prooticooccipitale zu den Skelettelementen der oben genannten großwüchsigen Bufo-Form. Dies lässt sich jedoch nicht mit Sicherheit feststellen.
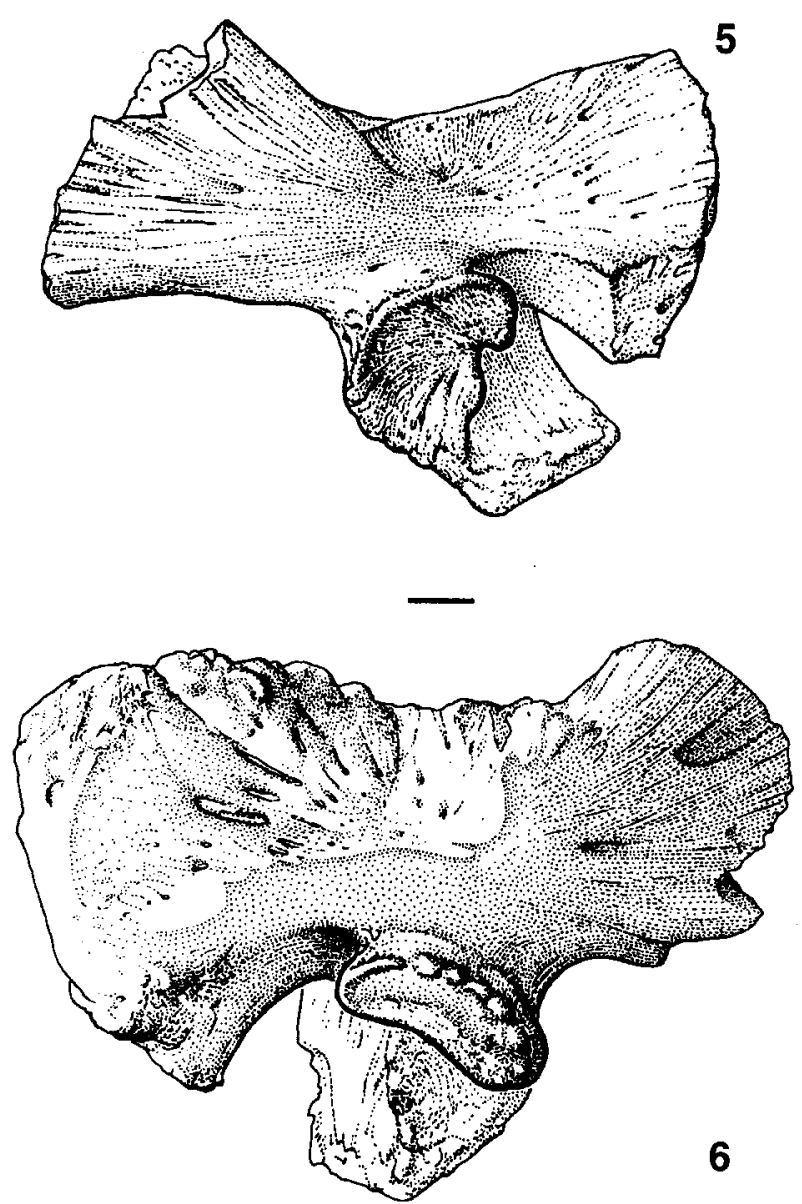

Abb. 5-6. 5. Bufo bufo, Scapula dex. (NHMS-MTe 976/7). 6 Bufo bufo subsp. indet., Scapula sin. (NHMS-MTe 976/3). Ansicht von lateral.

Figs 5-6. 5. Bufo bufo, right scapula (NHMS-MTe 976/7). 6. Bufo bufo subsp. indet., left scapula (NHMS-MTe 976/3). Lateral view. 
Ranidae Gray, 1825

\section{Rana temporaria Linnaeus, 1758}

Abb. 4, 10, 13

Zahlreiche Skelettelemente aus verschiedenen Fundkomplexen (NHMS-MTe 978-984)

Frontoparietale (Abb. 4) (26 sin., 19 dex.): wie bei der rezenten Art.

Maxillare mit Zähnen.

$S c$ apula (61): wie bei der rezenten Art.

Ilium (Abb. 13) (28 sin., 19 dex.): wie bei der rezenten Art.

Humerus $\delta^{*} \circ$ (38): wie bei der rezenten Art (Abb. 10 今).

Die Ausbildung der Frontoparietalia, der männlichen Humeri als auch der Ilia ist mit der von vergleichbaren Elementen der rezenten Art Rana temporaria identisch.

\section{cf. Salientia gen. et sp. indet.}

Abb. 3

Es liegen die Fragmente von drei fraglichen Parietale aus dem Fundkomplex Kals. 858 vor, die nicht zu Bufo gehören (NHMS-MTe 976/1). Ein derart gestaltetes Skelettelement ist bei Bufo nicht vorhanden. Davon gehören zwei der gleichen Seite und eins der entgegengesetzten Seite an. Eine Orientierung (rechts oder links) ist nicht mit Sicherheit vorzunehmen. Das paarige Parietale scheint nicht mit dem Frontale verwachsen zu sein. An den marginalen Begrenzungen der beiden vollständigen Fundstücke ist nur am vermutlichen processus lateralis eine Bruchkante zu erkennen. Die elliptische incrassatio frontoparietalis zeigt eine für Salientia typische Ausbildung (Špinar 1976). Die dorsale Oberfläche des relativ dickwandigen Elements ist leicht gewölbt und schwach runzelig (Abb. 3). Auf der ventralen Seite ist die vermutliche margo sagittalis leicht gefurcht. Eine generische bzw. spezifische Zuordnung kann derzeit nicht vorgenommen werden.

\section{Diskussion}

Die Amphibienfauna aus der oberpliozänen Erdfallfüllung von Kaltensundheim umfasst folgende Arten:
Bufo bufo (Linnaeus, 1758); Bufo bufo (Linnaeus, 1758) subsp.; Bufo sp.; Rana temporaria Linnaeus, 1758; Salientia gen. et spec. indet.

Mit Sicherheit sind demnach anhand der vorliegenden Skelettreste nur 2 Arten: Bufo bufo und Rana temporaria zu bestimmen, die jedoch auch gegenwärtig noch in Mitteleuropa verbreitet sind (Böhme 1977). Hinzu kommen Funde, die auf Arten bzw. Unterarten hindeuten, die noch weitgehend unbekannt sind und heute nicht mehr im Gebiet vorkommen. Die bedeutende Größe und andere Merkmale einiger Fundstücke veranlassten den Autor zunächst zu der Annahme, dass sich unter ihnen Skelettreste von Latonia befinden könnten. Er wurde darin in Diskussionen mit mehreren Fachkollegen bestärkt. Der bessere Kenntnisstand über Latonia seit der Neubeschreibung der Gattung durch Roček (1994) gab jedoch nun Gewissheit, dass $L a$ tonia nicht in der fossilen Herpetofauna von Kaltensundheim vertreten ist. Sie ist daher aus den bisherigen Faunenlisten zu streichen.

Latonia ist vor allem aus miozänen Fundstellen Europas bekannt (Hodrová 1987, Roček 1994, Sanchiz 1998, Špinar et al. 1993), wurde aber auch mehrfach aus Fundstellen pliozänen Alters angeführt (Sanchiz 1998).

Die Skelettreste von Bufo bufo aus den Füllsedimenten von Kaltensundheim zeigen einerseits große Übereinstimmung mit der rezent in Mitteleuropa verbreiteten Unterart Bufo bufo bufo (Linnaeus, 1758). Andererseits stehen diesen Funden einige Skelettelemente mit beachtlicher Größe gegenüber, die aber in der Merkmalsausbildung gleichfalls weitgehende Übereinstimmung mit denen von Bufo bufo haben. Diese deuten auf eine Bufo-Art hin, die durchaus die Dimensionen der mediterranen Unterart Bufo bufo spinosus (Daudin, 1803) erreichen konnte. Selbst bei großwüchsigen Exemplaren dieser Unterart ist jedoch die Dorsalseite des Frontoparietale nahezu völlig glatt. Auch bei der ähnlichen Bufo priscus Špinar, Klembara et Meszáro, 1993 aus dem mittleren Miozän von Devínska Nová Ves (Slovakia) ist die Dorsalfläche des Frontoparietale weitgehend glatt. Das kräftig ornamentierte Bufo-Frontoparietale ist daher auf eine andere, bisher nicht bekannte Bufo-Art zu beziehen. Da jedoch keine artikulierten Skelette dieser Art in den Füllsedimenten geborgen werden konnten, ist eine Zusammengehörigkeit dieser Elemente zu anderen Teilen des Skelettes zwar zu vermuten, aber nicht mit Sicherheit festzustellen. Von der Aufstellung einer nov. spec. wird daher Abstand genommen. Bisher sind im 


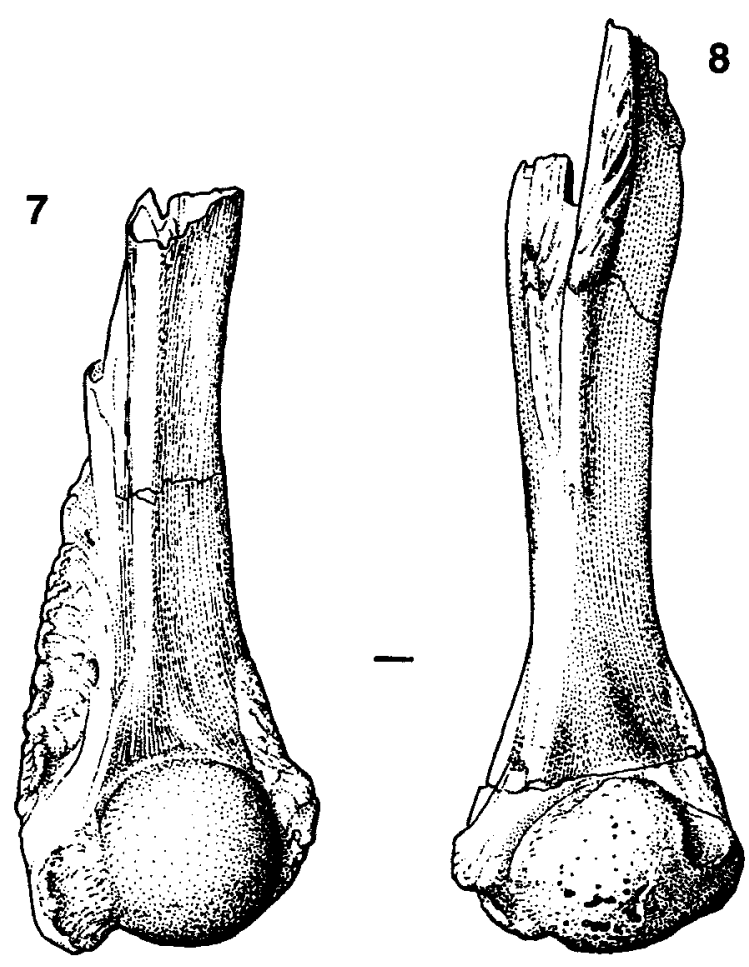

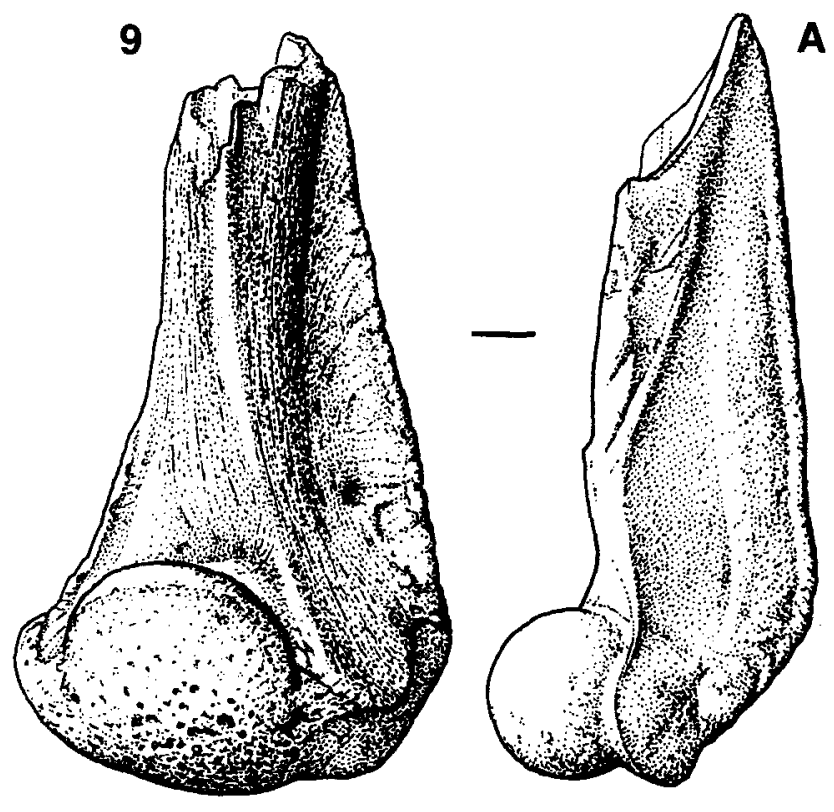

Pliozän Europas nur die auch rezent verbreiteten Arten Bufo bufo (Linnaeus, 1758), Bufo viridis Laurenti, 1768 und Bufo calamita Laurenti, 1768 bekannt (Sanchiz 1998).

Die Skelettelemente, die als Rana temporaria bestimmt werden, sind mit denen der rezenten Art völlig identisch. Dies betrifft sowohl die Ausbildung der skelettmorphologischen Merkmale als auch die Dimensionen. Körperproportionen konnten daraus nicht abgeleitet werden.

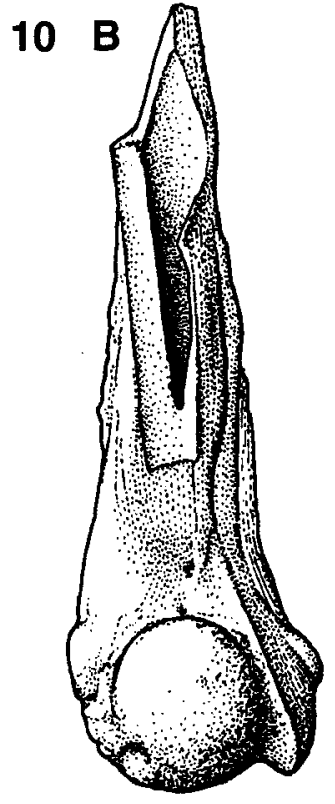

Abb. 7-10. 7. Bufo bufo subsp. indet., o Humerus sin. (NHMSMTe 976/4); 8. Bufo bufo subsp. indet., $q$ Humerus sin. (NHMSMTe 976/5); 9. Bufo bufo, o Humerus dex. (NHMS-MTe $976 / 8)$; 10. Rana temporaria, 0 Humerus dex. (NHMS-MTe 978/3); $\mathbf{A}$, von lateral; $\mathbf{B}$, von ventral.

Figs 7-10. 7. Bufo bufo subsp. indet., $\hat{\sigma}$ left humerus (NHMSMTe 976/4); 8. Bufo bufo subsp. indet., $\&$ left humerus (NHMSMTe 976/5); 9. Bufo bufo, of right humerus (NHMS-MTe 976/8); 10. Rana temporaria, right humerus (NHMS-MTe 978/3); A, lateral view; B, ventral view.

Die Funde von Kaltensundheim sind die bisher ältesten sicheren Nachweise dieser Art (Sanchiz 1998). Das aus den untermiozänen Dysodilen des Dietrichsberges bei Vacha von M. Böhme (2001) als Rana cf. temporaria beschriebene artikulierte Raniden-Skelett ist aufgrund des Erhaltungszustandes der Frontoparietalia nicht eindeutig zuzuordnen. Ob die aus dem Pliozän von Willershausen durch Špinar (1980) beschriebene Art Rana strausi mit Rana temporaria aus Kalten- 


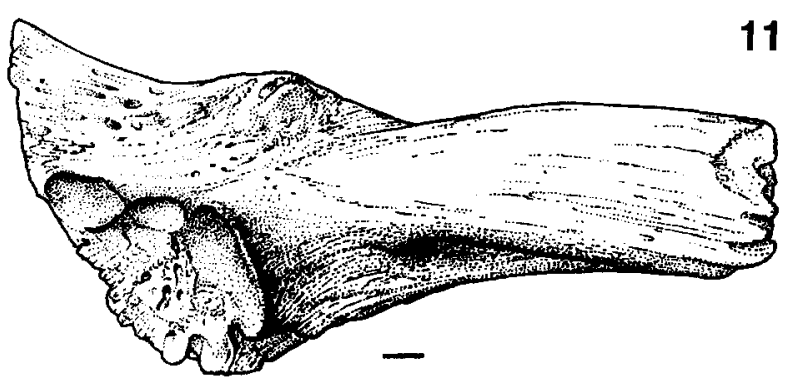

12
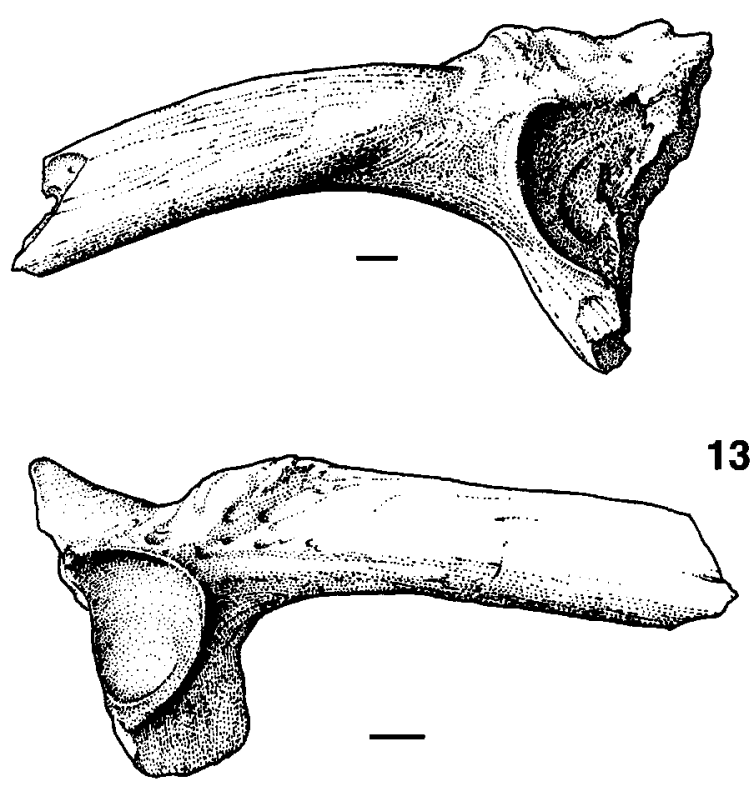

Abb.11-13. 11. Bufo bufo, Ilium dex. (NHMS-MTe 977/1); 12. Bufo bufo, Ilium sin. (NHMS-MTe $977 / 2$ ); 13. Rana temporaria, Ilium dex. (NHMS-MTe 978/2). Alle Ilia in lateraler Ansicht.

Figs 11-13. 11. Bufo bufo, right ilium (NHMS-MTe 977/1); 12. Bufo bufo, left ilium (NHMS-MTe 977/2); 13. Rana temporaria, right ilium (NHMS-MTe 978/2). Ilia in lateral view.

sundheim verglichen werden kann, muss offen bleiben, da in Kaltensundheim keine vollständigen artikulierten Skelette geborgen wurden.

Die als vermutliche Parietale (Abb. 3) definierten drei Fundstücke sind keine Skelettelemente von Bufo. Eine generische bzw. spezifische Zuordnung ist derzeit nicht möglich.

\section{Zur Paläoökologie}

Die Amphibienfauna von Kaltensundheim umfasst nur wenige, definierbare Arten. Mit Bufo bufo und Rana temporaria sind zwei Arten nachweisbar, die im Quartär Europas zahlreich überliefert und auch rezent noch häufig sind. Bufo bufo ist eine euryöke Form mit weiter Verbreitung (Engelmann et al. 1985, Günther 1996). Als Lebensraum bevorzugt sie lichte Wälder bis zum Offenland. Die rezente Form überwintert in frostfreien Verstecken im Boden. Zum Laichen werden überwiegend stehende Gewässer aufgesucht, wo die Laichschnüre an Pflanzenstengeln verankert werden. Während der Fortpflanzungszeit sind in Gewässern öfter Todesfälle kopulierender Paare zu beobachten.

Die rezente Rana temporaria ist in Europa und in West-Sibirien bis zum Ob weit verbreitet. Ihr Verbreitungsgebiet reicht von der subpolaren Tundra und Taiga bis zu mediterranen Bergwäldern. In Kaltensundheim erscheint die Art als Bestandteil einer temperierten Waldfauna. Unter den europäischen Salientia hat diese Art die größte Temperaturtoleranz. Im Pleistozän ist $R a$ na temporaria selbst in hochglazialen Abschnitten noch nachweisbar (Böhme 2000). Die Häufigkeit der Funde in den Füllsedimenten des fossilen Erdfalls von Kaltensundheim und die Überlieferung von artikulierten Teilskeletten legen nahe, dass es sich möglicherweise um Individuen handelt, die Ruhephasen (Winterruhe ?) im Schlamm des Gewässergrundes verbrachten und dabei umkamen. Im rezenten Verbreitungsgebiet überwintert die Art im Schlamm von Gewässern oder in Höhlungen im Boden (Günther 1996). Dabei kann es zu Massenansammlungen überwinternder Tiere kommen. Sauerstoffmangel am Gewässergrund führt dann gelegentlich zu einer erheblichen Mortalität. Die Eiablage sowie die Larvenentwicklung erfolgt in stehenden, allenfalls langsam fließenden Gewässern. Der pliozäne Erdfallsee von Kaltensundheim gehörte offensichtlich zum unmittelbaren Lebensraum dieser Art.

Die in der Fundstelle Kaltensundheim belegte großwüchsige Bufo-Form, aus der man für die Zeit der Sedimentation auf mediterrane Klimabedingungen schließen könnte, steht zu dem auf der Grundlage der Pflanzenreste rekonstruierten Klimacharakter im Gegensatz. Vergleichbare Probleme bereitet auch die ökologische Interpretation der in den Fundschichten überlieferten Mastodonten (Mammut borsoni). Offenbar sind unsere Kenntnisse über die Zusammensetzung pliozäner Faunen und Floren noch nicht ausreichend, um solche Gegensätze interpretieren zu können.

\section{Danksagung}

Der Verfasser hat Herrn J. Mendau für die Anfertigung und Herrn Dr. R. Schoch (beide Museum für Naturkunde der Humboldt-Universität zu Berlin, Institut für Paläontologie) für die datentechnische Verarbeitung der Zeichnungen herzlich zu danken. 


\section{Literatur}

Böhme, G. 1963. Über den Skelettfund eines Pliocerviden aus dem Pliozän von Kaltensundheim/Rhön. - Paläontologische Abhandlungen 1 (4): 353-372.

- 1968. Pliozäne und pleistozäne Reliefentwicklung und die Plio - Pleistozän Grenze in der östlichen Vorderrhön. Unveröff Diplomarbeit, Fachrichtung Geologie der Humboldt-Universität zu Berlin, Maschschr. 107 S., 41 Abb., 13 Anl.

- 1977. Zur Bestimmung quartärer Anuren Europas an Hand von Skelettelementen. - Wissenschaftliche Zeitschrift Humboldt-Universität zu Berlin, MathematischNaturwissenschaftliche Reihe 26 (3): 283-300.

- 1982. Biometrische Untersuchungen an Skelettelementen von Anuren. - Wissenschaftliche Zeitschrift HumboldtUniversität zu Berlin, Mathematisch-Naturwissenschaftliche Reihe 31 (3): 209-216.

- 1992. Pliozäne Erdfallbildung in der östlichen Vorderrhön und ihre Bedeutung für die Morphogenese des Gebietes. - Zeitschrift für geologische Wissenschaften 20 (5/6): 447-454.

- 2000. Fossile Amphibien und Reptilien im Quartär Thüringens. - Veröffentlichungen des Naturkundemuseums Erfurt 19: 79-97.

Böhme, M. 2001. The oldest representative of a brown frog (Ranidae) from the Early Miocene of Germany. - Acta Palaeontologica Polonica 46 (1): 119-124.

Engelmann, W.-E., Fritzsche, J., Günther, R. \& Obst, F. J. 1985. Lurche und Kriechtiere Europas. - Neumann Verlag Leipzig, Radebeul.

Günther, R. (Hrg.) 1996. Die Amphibien und Reptilien Deutschlands. - G. Fischer Verlag Jena, Stuttgart, Lübeck, Ulm.
Hodrová, M. 1987. Lower Miocene frogs from the Dolnice locality in the Cheb basin (Czechoslovakia). - Acta Universitatis Carolinae - Geologica 2: 97-115.

Krutzsch, W. 1988. Kritische Bemerkungen zur Palynologie und zur klimastratigraphischen Gliederung des Pliozäns bis tieferen Altpleistozäns in Süd-, Südwest-, Nordwestund pro parte Mitteleuropa sowie die Lage der Plio-Pleistozän-Grenze in diesem Gebiet. - Quartärpaläontologie 7: 7-51.

Mai, D. \& Walther, H. 1988. Die pliozänen Floren von Thüringen, Deutsche Demokratische Republik. - Quartärpaläontologie 7: 55-297.

Roček, Z. 1994. Taxonomy and distribution of Tertiary discoglossid (Anura) of the genus Latonia v. MEYER, 1843. Geobios 27 (6): 717-751.

Sanchiz, F. B. 1977. La Familia Bufonidae (Amphibia, Anura) en el Terciario Europeo. - Trabajos neógeno-cuarternario, Instituto "Lucas Mallada" (CSIC) 8: 75-111.

- 1998. Salientia. In Wellnhofer, P. (ed.). Handbuch der Paläoherpetologie, Teil 4: I-XII: 1-275. Verlag Dr. F. Pfeil, München.

Schaarschmidt, F. 1958. Fund eines Mastodon in der Rhön. - Neue Museumskunde 1: 290-292.

Špinar, Z. 1976. Endolymphatic sacs and dorsal endocranial pattern: Their significance for systematics and phylogeny of frogs. - Vestnik Ústředního Ústavu Geologického 51: 285-290.

- 1980. Fossile Raniden aus dem oberen Pliozän von Willershausen (Niedersachsen). - Stuttgarter Beiträge zur Naturkunde, Serie B (Geologie Paläontologie) 53: 1-53.

Špinar, Z., Klembara, J. \& Meszáro, S. 1993. A new toad from the Miocene at Devínska Nová Ves (Slovakia). Západné Karpaty Séria Paleontológia 17: 135-160. 